\title{
VARIETIES OF RINGS WITH DEFINABLE PRINCIPAL CONGRUENCES
}

\author{
G. E. SIMONS
}

\begin{abstract}
A variety $\mathcal{T}$ of rings has definable principal congruences (DPC) if there is a first order sentence defining principal two-sided ideals for all rings in $\mathfrak{T}$. The key result is that for any ring $R, V\left(M_{n}(R)\right)$ does not have DPC if $n \geqslant 2$. This allows us to show that if $V(R)$ has DPC, then $R$ is a polynomial identity ring. Results from the theory of PI rings are used to prove that for a semiprime ring $R, V(R)$ has DPC if and only if $R$ is commutative. An example of a finite, local, noncommutative ring $R$ with $V(R)$ having DPC is given.
\end{abstract}

The concept of definable principal congruences was introduced by Baldwin and Berman [2] in a study of the size of subdirectly irreducible algebras in a variety. Earlier, Taylor [14] had shown that a variety is residually small (has an upper bound on the size of its subdirectly irreducible algebras) if and only if every subdirectly irreducible algebra in the variety has power $\leqslant 2^{\eta}$, where $\eta=\max \left(\boldsymbol{\aleph}_{0}\right.$, number of fundamental operations of the algebras in the variety). Baldwin and Berman [2] showed that if a variety has definable principal congruence, then it is residually small if and only if there is an integer $N$ such that each subdirectly irreducible algebra in the variety has power $<N$. They asked if a variety generated by a finite algebra always has definable principal congruences. Burris [3] gave an example showing that this is not true in general.

McKenzie [12] showed that there is a relationship between definable principal congruences and finite axiomatizability: a variety with definable principal congruences having a finite bound on the size of its subdirectly irreducible algebras is finitely axiomatizable. This result was used to show that paraprimal varieties are finitely axiomatizable. McKenzie also showed that the only varieties of lattices with definable principal congruences are the varieties of distributive lattices. He stated that the question of whether varieties generated by a finite group or ring have definable principal congruences was open.

Burris and Lawrence $[4,5]$ gave an example of a finite ring generating a variety without definable principal congruences and obtained some results on finite groups generating varieties with definable principal congruences. They showed that if $G$ is a finite group and $V(G)$ has definable principal congruences, then $G$ is nilpotent. Baker [1] solved the problem completely for finite groups by showing that a locally

Received by the editors June 24, 1982.

1980 Mathematics Subject Classification. Primary 16A38, 08B99; Secondary 16A12, 16A70, 08B05.

$\mathrm{Key}$ words and phrases. Varieties of rings, definable principal congruences, polynomial identity rings. 
finite variety of groups has definable principal congruences if and only if it is generated by a finite group satisfying the commutator identity $[x, y, x]=1$.

This paper is concerned with the characterization of rings that generate varieties with definable principal congruences. We first show that if $A$ is an algebra over a field and $V(A)$ has definable principal congruences, then every idempotent of $A$ must be central. It follows that $V\left(M_{n}(R)\right)$ does not have definable principal congruences if $n \geqslant 2$. Next we show that if $R$ is a ring generating a variety with definable principal congruences, then $R$ satisfies a polynomial identity. Using some of the major results about polynomial identity rings (PI rings) we show that a semiprime ring $R$ generates a variety with definable principal congruences if and only if $R$ is commutative. Finally, we conclude with an example of a finite noncommutative ring that generates a variety with definable principal congruences.

For the basic facts about varieties see [6,7 or 9]. Lambek [11] treats the basic material on rings and Rowen [13] gives a comprehensive treatment of PI rings.

Definition [2]. A variety $\mathcal{W}$ of algebras has definable principal congruences (DPC) if and only if there is a first order sentence $\Phi(a, b, c, d)$ such that for all $A \in \mathcal{V}$ and for all $a, b, c, d \in A,(a, b) \in \theta(c, d) \Leftrightarrow \Phi(a, b, c, d)$, where $\theta(c, d)$ is the principal congruence generated by $(c, d)$.

Throughout this paper we use the language $L=\{0,1,+,-, \cdot\}$ of rings with identity. Thus if $\mathcal{V}$ is a variety of rings then $\mathcal{V}$ has DPC if and only if there is a first order sentence $\Phi(x, y)$ in $L$ such that for all rings $R \in \mathcal{V}$ and for all $x, y \in R$, $x \in R y R \Leftrightarrow \Phi(x, y)$, where $R y R$ is the two sided ideal of $R$ generated by $y$.

LEMMA $1([4,5])$. If $\mathscr{K}$ is a class of rings, then $V(\mathcal{K})$ has DPC if and only if there are integers $n$ and $k$ with $n>k \geqslant 1$ and polynomials $r_{i}\left(y, x_{1}, \ldots, x_{n}, z_{1}, \ldots, z_{n}\right)$, $s_{i}\left(y, x_{1}, \ldots, x_{n}, z_{1}, \ldots, z_{n}\right), 1 \leqslant i \leqslant k$, such that

$$
\mathscr{\gamma}_{i} \vDash \sum_{i=1}^{n} x_{i} y z_{i}=\sum_{i=1}^{k} r_{i}(y, \bar{x}, \bar{z}) y s_{i}(y, \bar{x}, \bar{z})
$$

where $\bar{x}=\left(x_{1}, \ldots, x_{n}\right), \bar{z}=\left(z_{1}, \ldots, z_{n}\right)$.

Somewhat roughly, this means that $V(\mathcal{K})$ has DPC if and only if, for all rings $R \in V(\mathcal{K})$ and for all $y \in R$, given an element of $R y R$ formed in $n$ "steps" there is a specified way of producing that element in fewer "steps", where a "step" consists of forming the product $a y b$ with $a, b \in R$ and adding this product to the result of the previous steps. Repeated application of this procedure shows that every element of $R y R$ can be obtained in at most $k$ steps, for all $R \in V(\mathscr{K})$ and all $y \in R$.

LEMMA 2. The variety $\mathcal{T}$ of all commutative rings has DPC.

Proof. $\mathcal{T} \vDash \sum_{i=1}^{2} x_{i} y z_{i}=1 \cdot y \cdot \sum_{i=1}^{2} x_{i} z_{i}$, so Lemma 1 shows that $\mathcal{V}$ has DPC.

Since any subvariety of a variety with DPC itself has DPC, Lemma 2 shows that the variety generated by any commutative ring has DPC.

TheOREM 3. Let $A$ be an algebra over a field $F$. If $V(A)$ has $D P C$, then every idempotent of $A$ is central. 
Proof. Suppose $A$ has a noncentral idempotent $e$. Then there is $a \in A$ such that either $e a(1-e) \neq 0$ or $(1-e) a e \neq 0$. Assume $e a(1-e) \neq 0$. If $V(A)$ has DPC, then by Lemma $1, A$ satisfies an equation

$$
\sum_{i=1}^{n} x_{i} y z_{i}=\sum_{i=1}^{k} r_{i}(y, \bar{x}, \bar{z}) y s_{i}(y, \bar{x}, \bar{z})
$$

where $n>k \geqslant 1$ and $r_{i}, s_{i}$ are polynomials in $y, \bar{x}, \bar{z}$ with integer coefficients. Also any ring $T \in V(A)$ must satisfy this equation.

Suppose there is a ring $T \in V(A)$ with elements $a_{i}, b, c_{i}, 1 \leqslant i \leqslant n$, such that (i) $b^{2}=0$, (ii) $b a_{i}=c_{i} b=0,1 \leqslant i \leqslant n$, and (iii) any two elements of $\left\{a_{i}, c_{j} ; 1 \leqslant i, j \leqslant\right.$ $n$ ) commute. Substituting $a_{i}$ for $x_{i}, b$ for $y$ and $c_{i}$ for $z_{i}$ in (1), we see that the only nonzero terms of the r.h.s. of (1) come from monomials of the $r_{i}$ 's without any $y$ or $z_{i}$ 's in them and monomials of the $s_{i}$ 's without any $y$ or $x_{i}$ 's in them. Thus we have

$$
\sum_{i=1}^{n} a_{i} b c_{i}=\sum_{i=1}^{k}\left(\lambda_{i}+\sum_{j} \sigma_{i j}\right) b\left(\tau_{i}+\sum_{j} \phi_{i j}\right), \quad \text { where } \lambda_{i}, \tau_{i} \in \mathbf{Z},
$$

$\sigma_{i j}$ is a product of $a_{i}$ 's, $\phi_{i j}$ a product of $c_{i}$ 's.

Since the conditions (i), (ii), (iii) are met if each $a_{i}=0$, or each $c_{i}=0$ or $a_{i}=c_{i}=0,1 \leqslant i \leqslant n$, we can make these substitutions to obtain

$$
0=\sum_{i=1}^{k} \lambda_{i} b\left(\tau_{i}+\sum \phi_{i j}\right)=\sum_{i=1}^{k}\left(\lambda_{i}+\sum \sigma_{i j}\right) b \tau_{i}=\sum_{i=1}^{k} \lambda_{i} b \tau_{i} .
$$

From these, we get the equation

$$
\sum_{i=1}^{n} a_{i} b c_{i}=\sum_{i=1}^{k}\left(\sum_{j} \sigma_{i j}\right) b\left(\sum_{j} \phi_{i j}\right) \text {. }
$$

Now, choose $2 n$ distinct primes $p_{1}, \ldots, p_{n}, q_{1}, \ldots, q_{n}$ and define elements $a_{i}$, $b, c_{i} \in \prod_{j=1}^{\infty} A \in V(A), 1 \leqslant i \leqslant n$, by

$$
a_{i}(j)=\left\{\begin{array}{ll}
e, & p_{i} \mid j, \\
0, & p_{i} \nmid j,
\end{array} \quad b(j)=e a(1-e), \quad c_{i}(j)= \begin{cases}1-e, & q_{i} \mid j, \\
0, & q_{i} \nmid j .\end{cases}\right.
$$

By assumption, $b \neq 0$ and it is clear that these elements satisfy conditions (i), (ii), (iii) above. Since each $a_{i}$ and $c_{i}$ is idempotent we can write (3) in the following form:

$$
\sum_{i=1}^{n} a_{i} b c_{i}=\sum_{i=1}^{k}\left(\sum_{j} \mu_{i j} a_{1}^{j_{1}} \cdots a_{n}^{j_{n}}\right) b\left(\sum_{l} \nu_{i l} c_{1}^{l_{1}} \cdots c_{n}^{l_{1}}\right)
$$

where $\mu_{i j}, \nu_{i l} \in \mathbf{Z}, \sum_{i=1}^{n} j_{i} \neq 0, \sum_{i=1}^{n} l_{i} \neq 0$ for each $j$ and $l$, and $j_{i}, l_{i} \in\{0,1\}$.

Let $s$ be the sum of the terms on the r.h.s. of (4) of the form $\mu a_{j} b c_{k}, 1 \leqslant j, k \leqslant n$, $\mu \in \mathbf{Z}$. Then $\sum_{i=1}^{n} a_{i} b c_{i}-s=t$, where $t$ is the sum of the terms on the r.h.s. of (4) which contain at least two $a_{i}$ 's or two $c_{j}$ 's. Thus $t\left(p_{i} q_{j}\right)=0$ for $1 \leqslant i, j \leqslant n$ so $\left(\sum_{i=1}^{n} a_{i} b c_{i}-s\right)\left(p_{i} q_{j}\right)=0$ for $1 \leqslant i, j \leqslant n$. Then $\sum_{i=1}^{n} a_{i} b c_{i}=s$ since for every $m$ such that $p_{i} q_{j} \nmid m$ for $1 \leqslant i, j \leqslant n,\left(\sum_{i=1}^{n} a_{i} b c_{i}-s\right)(m)=0$. Therefore $\sum_{i=1}^{n} a_{i} b c_{i}=$ $\sum_{i=1}^{n}\left(\sum_{j=1}^{n} \mu_{i j} a_{j}\right) b\left(\sum_{m=1}^{n} \nu_{i m} c_{m}\right)=\sum_{i=1}^{n}\left(\sum_{j=1}^{k} \nu_{j i}\left(\sum_{m=1}^{n} \mu_{j m} a_{m}\right)\right) b c_{i}, \nu_{i j}, \mu_{i j} \in \mathbf{Z}$. Let $w_{j}$ $=\sum_{m=1}^{n} \mu_{j m} a_{m}, 1 \leqslant j \leqslant k$, so $\sum_{i=1}^{n}\left(a_{i}-\sum_{j=1}^{k} \nu_{j i} w_{j}\right) b c_{i}=0$. Considering the $p_{m} q_{i}$ coordinates for $i$ fixed, $1 \leqslant m \leqslant n$, we see that $\left(a_{i}-\sum_{j=1}^{k} \nu_{j i} w_{j}\right) b c_{i}=0$ for $1 \leqslant i \leqslant n$. For 
each $i, a_{i}-\sum_{j=1}^{k} \nu_{j i} w_{j}=\sum_{j=1}^{n} \gamma_{j} a_{j}, \gamma_{j} \in \mathbf{Z} .\left(\sum_{j=1}^{n} \gamma_{j} a_{j}\right) b c_{i}=0$ so $\gamma_{j} a_{j} b c_{i}\left(p_{j} q_{i}\right)=0$, hence $\gamma_{j} e a(1-e)=0, \gamma_{j} \in \mathbf{Z}$. Then $\gamma_{j} \cdot 1=0$, since $\gamma_{j} \in F$ and $e a(1-e) \neq 0$. Therefore, for each $i$ we have $a_{i}=\sum_{j=1}^{k} \nu_{j i} w_{j}$.

Let $S$ be the subring of $\prod_{j=1}^{\infty} A$ generated by 1 . If $\sum_{i=1}^{n} s_{i} a_{i}=0, s_{i} \in S$, then $s_{i} e\left(\sum s_{j} a_{j}\right)\left(p_{i}\right)=0$, so $s_{i} \cdot 1=0$. Let $M$ be the free $S$ module with free basis $a_{1}, \ldots, a_{n}$ and let $W$ be the $S$ module generated by $\left\{w_{1}, \ldots, w_{k}\right\}$. By definition each $w_{i} \in M$ and we have shown each $a_{i} \in W$, so $M=W . S$ is commutative, hence has invariant basis number (IBN), so $n=\operatorname{rank} M=\operatorname{rank} W \leqslant k$, contradicting $n>k$.

COROllaRY 4. Let $A$ be a semiperfect algebra over a field. If $V(A)$ has DPC, then $A$ is isomorphic to a finite direct product of local algebras over $F$.

Proof. Any semiperfect ring has a finite orthogonal set of local idempotents $e_{1}, \ldots, e_{n}$ with $e_{1}+e_{2}+\cdots+e_{n}=1$ [11, Corollary 2, p. 76]. Since $V(A)$ has DPC, Theorem 3 shows that each $e_{i}$ is central. Therefore $A \cong \prod_{i=1}^{n} e_{i} A$ with $e_{i} A$ a local algebra over $F$.

THEOREM 5. Let $R$ be a ring, $n$ an integer, $n \geqslant 2$. Then $V\left(M_{n}(R)\right)$ does not have $D P C$.

Proof. Let $S$ be the subring of $R$ generated by 1 . Then $S \cong \mathbf{Z} / k \mathbf{Z}$ for some $k \geqslant 0$, so there is a prime $p$ such that $\mathbf{Z} / p \mathbf{Z} \in V(R) . M_{n}(S) \in V\left(M_{n}(R)\right)$, hence $M_{n}(\mathbf{Z} / p \mathbf{Z}) \in V\left(M_{n}(R)\right)$. Since $n \geqslant 2, M_{n}(\mathbf{Z} / p \mathbf{Z})$ has a noncentral idempotent, so by Theorem $3 V\left(M_{n}(\mathbf{Z} / p \mathbf{Z})\right)$ does not have DPC. Therefore $V\left(M_{n}(R)\right)$ does not have DPC.

COROllaRY 6. Let $R=\left(\begin{array}{l}\mathbf{z}_{2} \\ 0_{2} \\ \mathbf{z}_{2}\end{array}\right)$. Then $V(R)$ does not have DPC and $R$ is the smallest ring with this property.

Proof. Since $R$ is an algebra over $\mathbf{Z}_{2}$ with a noncentral idempotent, $V(R)$ does not have DPC, by Theorem 3. Any ring $T$ with fewer than 8 elements must be commutative [8] and so Lemma 2 shows that $V(T)$ has DPC. Note that $R$ is (up to isomorphism) the only noncommutative ring with 8 elements [8].

Definition ([13]). Let $f\left(x_{1}, \ldots, x_{n}\right)$ be a polynomial with integer coefficients in noncommuting indeterminates. If $f\left(r_{1}, \ldots, r_{n}\right)=0$ for all choices of $r_{1}, \ldots, r_{n}$ in a ring $R$, then $f$ is called an identity of $R$. An identity of $R$ with a coefficient $\alpha$ such that $\alpha \cdot 1 \neq 0$ is called an $R$-proper identity. If a ring $R$ has an identity which is multilinear and has 1 as a coefficient, then $R$ is called a polynomial identity ring (PI ring).

TheOREM 7. Let $R$ be a ring such that $V(R)$ has DPC. Then $R$ is a PI ring.

Proof. By Lemma 1 there are integers $n$ and $k$ with $n>k \geqslant 1$ and polynomials $r_{i}$, $s_{i}, 1 \leqslant i \leqslant k$, with integer coefficients such that $R$ satisfies the identity

$$
\sum_{i=1}^{n} x_{i} y z_{i}=\sum_{i=1}^{k} r_{i}(y, \bar{x}, \bar{z}) y s_{i}(y, \bar{x}, \bar{z}) .
$$

By [13, Theorem 1.6.46], $R$ is a PI ring if this identity is $\bar{R}$ proper for every nonzero homomorphic image $\bar{R}$ of $R$. Let $I$ be the ideal of $R$ generated by the coefficients of 
the identity. If $I \neq R$ then every coefficient annihilates $R / I$ and so the identity would be an identity of $M_{n}(R / I)$ for all integers $n$, contradicting Theorem 5 . Therefore $I=R$ and the identity is $\bar{R}$ proper for all nonzero homomorphic images of $R$. Thus $R$ is a PI ring.

THEOREM (KAPLANSKY, [13, THEOREM 1.5.16]). Let $R$ be a primitive ring satisfying an $R$-proper identity of degree $d$. Then $R \cong M_{k}(D)$ where $D$ is a division algebra and $[R: Z(R)]=k^{2}[D: Z(D)]=n^{2}$ with $2 n \leqslant d$.

LEMMA 8. Let $R$ be a primitive ring. If $V(R)$ has DPC, then $R$ is a division algebra and $[R: Z(R)]$ is finite.

Proof. By Theorem 5 and Kaplansky's theorem, $R \cong M_{k}(D)$ and $[R: Z(R)]$ is finite. Theorem 5 shows that $k=1$, so $R$ is isomorphic to a division algebra $D$.

LEMMA 9 ([13, EXERCISE 2.3.4, p. 149]). Let $F$ be an infinite field, $R$ and $F$ algebra, $S$ a commutative $F$ algebra. Then every identity of $R$ is an identity of $R \otimes_{F} S$.

THEOREM 10. Let $R$ be a primitive ring. Then $V(R)$ has DPC if and only if $R$ is a field.

PRoof. If $R$ is a field, Lemma 2 shows that $V(R)$ has DPC. For the converse we consider two cases:

(i) $Z(R)$ is finite. By Lemma $8[R: Z(R)]$ is finite so $R$ is a finite division algebra and thus a field.

(ii) $Z(R)$ is infinite. Lemma 8 shows that $Z(R)$ is a field $F$. Let $A$ be an algebraic closure of $F$. Then $R \otimes_{F} A$ is simple and $\left[R \otimes_{F} A: A\right]=[R: Z(R)]=n^{2}$. Since $R \otimes_{F} A$ is a finite-dimensional simple algebra and $A$ is algebraically closed, $R \otimes_{F} A \cong M_{n}(A)$. Lemma 9 shows that $R \otimes_{F} A$ satisfies all the identities of $R$, so $V\left(R \otimes_{F} A\right)$ has DPC since $V(R)$ has DPC. By Theorem $5, V\left(M_{n}(A)\right)$ has DPC implies that $n=1$. Since $[R: Z(R)]=1$, Lemma 8 shows that $R$ is a field.

Theorem (Posner-Rowen et Al., [10, Theorem 2, p. 57]). Let $R$ be a prime PI ring and let $R_{Z}$ be the localization of $R$ at its centre $Z(R)$. Then $R_{Z} \cong M_{n}(D)$ where $D$ is a finite-dimensional division algebra, $R$ is a subring of $R_{Z}$ and $R$ and $R_{Z}$ satisfy the same polynomial identities.

THEOREM 11. Let $R$ be a prime ring. Then $V(R)$ has DPC if and only if $R$ is commutative.

Proof. If $R$ is commutative, then Lemma 2 shows that $V(R)$ has DPC. If $V(R)$ has DPC then the Theorem of Posner-Rowen shows that $V\left(R_{Z}\right)$ has DPC and Theorem 10 shows that $R_{Z}$ is a field. Since $R$ is a subring of $R_{Z}, R$ must be commutative.

COROLlaRY 12. Let $R$ be a semiprime ring. Then $V(R)$ has DPC if and only if $R$ is commutative.

Proof. If $R$ is commutative, Lemma 2 shows that $V(R)$ has DPC. Conversely, since $R$ is semiprime it is a subring of the ring $\prod_{i \in I} R / P_{i},\left\{P_{i}: i \in I\right\}$ being the set of 
prime ideals of $R$. Each $R / P_{i}$ is a prime ring and $V\left(R / P_{i}\right)$ has DPC since $V\left(R / P_{i}\right) \subseteq V(R)$, so Theorem 11 shows that each $R / P_{i}$ is commutative. Thus $R$ is commutative.

While sufficiently nice rings must be commutative to generate varieties with DPC, there are many examples of noncommutative rings that generate varieties with DPC.

The following example is due to John Lawrence. It is a finite local noncommutative ring that generates a variety with DPC (cf. Corollary 4).

TheOREM 13 (J. LAWRENCE). Let $R=(Z / p Z)[X, Y] /\left\langle\left\{X, Y^{3}\right\}\right\rangle$, where $p$ is a prime and $X, Y$ are noncommuting indeterminates. Then $R$ is a finite local noncommutative ring and $V(R)$ has $D P C$.

Proof. The Jacobson radical of $R, J(R)$, is clearly $\langle X, Y\rangle . R / J(R) \cong \mathbf{Z} / p \mathbf{Z}$ so $R$ is local. It is clearly finite and noncommutative since $X Y-Y X \notin\left\langle\{X, Y\}^{3}\right\rangle$. Note that $J(R)^{3}=0$. Any element $r \in R$ can be written as $f+j$ with $f \in \mathbf{Z} / p \mathbf{Z}$ and $j \in J(R)$. Choose $k$ so that $p^{k} \geqslant 3$ and let $q=p^{k}$. Then $r^{q}=(f+j)^{q}=f^{q}+j^{q}=f$ since $j^{q} \in J(R)^{q}=0$ and $f^{q}=f$. Thus for each $r \in R, r^{q} \in Z(R)$ and $r-r^{q} \in$ $J(R)$. For $r, s, t \in R,\left(r-r^{q}\right)\left(s-s^{q}\right)\left(t-t^{q}\right) \in J(R)^{3}=0$. Since $r^{q}, s^{q}, t^{q} \in Z(R)$ we can rewrite this in the form $r s t=s \alpha(r, s, t)+\beta(r, s, t) s$, where $\alpha$ and $\beta$ are polynomials in $r, s, t$ with integer coefficients. Then

$$
\sum_{i=1}^{3} r_{i} s t_{i}=s\left(\sum_{i=1}^{3} \alpha\left(r_{i}, s, t_{i}\right)\right)+\left(\sum_{i=1}^{3} \beta\left(r_{i}, s, t_{i}\right)\right) s
$$

for all $r_{1}, r_{2}, r_{3}, s, t_{1}, t_{2}, t_{3} \in R$. By Lemma $1, V(R)$ has DPC.

ACKNOWLEDGEMENTS. I would like to thank Dr. John Lawrence for many helpful conversations on this problem and for permission to include the above example.

\section{REFERENCES}

1. K. A. Baker, Definable normal closures in locally finite varieties of groups, Houston J. Math. 7 (1981), 467-471.

2. J. T. Baldwin and J. Berman, The number of subdirectly irreducible algebras in a variety, Algebra Universalis 5 (1979), 379-389.

3. S. Burris, An example concerning definable principal congruences, Algebra Universalis 7 (1977), 403-404.

4. S. Burris and J. Lawrence, Definable principal congruences in varieties of groups and rings, Algebra Universalis 9 (1979), 152-164.

5. A A correction to [4], Algebra Universalis 13 (1981), 264-267.

6. S. Burris and H. P. Sankappanavar, $A$ course in universal algebra, Springer-Verlag, New York, 1981.

7. P. M. Cohn, Universal algebra, rev. ed., Reidel, Dordrecht, 1981.

8. K. E. Eldridge, Orders for finite non-commutative rings with unity, Amer. Math. Monthly 75 (1968), $512-514$

9. G. Gratzer, Universal algebra, 2nd ed., Springer-Verlag, New York, 1979.

10. N. Jacobson, PI-algebras, Lecture Notes in Math., vol. 441, Springer-Verlag, New York, 1975.

11. J. Lambek, Lectures on rings and modules, Chelsea, New York, 1976.

12. R. McKenzie, Paraprimal varieties: A study of finite axiomatizability and definable principal congruences in locally finite varieties, Algebra Universalis 8 (1978), 336-348.

13. L. H. Rowen, Polynomial identities in ring theory, Academic Press, New York, 1980.

14. W. Taylor, Residually small varieties, Algebra Universalis 2 (1972), 33-53.

Department of Pure Mathematics, University of Waterloo, Waterloo, Ontario, Canada N2L 